\title{
Polarization dependence of electroluminescence from closely-stacked and columnar quantum dots
}

\author{
Philipp Ridha · Lianhe Li · Marco Rossetti • \\ Gilles Patriarche · Andrea Fiore
}

Received: 27 June 2007 / Accepted: 12 December 2007 / Published online: 12 January 2008

C) Springer Science+Business Media, LLC. 2008

\begin{abstract}
Quantum dots (QDs) have a potential for application in semiconductor optical amplifiers (SOAs), due to their high saturation power related to the low differential gain, fast gain recovery and wide gain spectrum compared to quantum wells. Besides all advantages, QDs realized by Stranski-Krastanov growth mode have a flat shape which leads to a gain anisotropy and a related transverse magnetic (TM) and -electric (TE) polarization dependence as compared to bulk material. This has so far prevented their applications in SOAs. It has been suggested that control of optical polarization anisotropy of the QD can be obtained through QD shape engineering, in closely stacked or columnar QDs (CQDs). To this aim, we have fabricated and tested SOA structures based on closely-stacked and columnar QDs. Closely-stacked InAs QDs with 4, 6 and $10 \mathrm{~nm}$ GaAs spacer showed a minor improvement in the ratio of TM and TE integrated electroluminescence (EL) over standard QDs along with a strong reduction in efficiency. In contrast, a large improvement was obtained in CQDs, depending on the number of stacked submonolayers which can be attributed to the more symmetric shape of columnar QDs. A relatively small spectral separation $(\Delta E \sim 21 \mathrm{meV})$ between TE- and TM-EL peaks has been observed showing that heavy- and light hole-like states, respectively are energetically close in these QDs. These results indicate that columnar QDs have a significant potential for polarization-independent QD SOA.
\end{abstract}

Keywords Quantum dots · Semiconductor optical amplifiers

P. Ridha $(\varangle) \cdot$ L. Li · M. Rossetti · A. Fiore

Ecole Polytechnique Federale de Lausanne (EPFL), 1015 Lausanne, Switzerland

e-mail: philipp.ridha@epfl.ch

G. Patriarche

Laboratory for Photonics and Nanostructures (LPN), Centre National de la Recherche Scientifique (CNRS), 91460 Marcoussis, France 


\section{Introduction}

Quantum dots (QDs) have a potential for application in semiconductor optical amplifiers (SOAs), due to their high saturation power related to the low differential gain, fast gain recovery and wide gain spectrum compared to quantum wells (QWs) (Sugawara et al. 2001; Berg et al. 2003; Akiyama et al. 2005). However, polarisation-independence is also needed for in-line amplifier applications. Due to their flat shape and quasi-biaxial compressive strain, self-assembled quantum dots have a valence-band ground state of the heavy-hole type, which does not couple to light polarized along the growth axis, i.e. transverse magnetic (TM) mode in a guided-wave configuration. A hope exists, however, that both the shape and the strain distribution can be changed, either by varying the QD height/diameter ratio, e.g. by the growth of the capping layer or changing the growth mode of the QD, or by using stacks of closelyspaced QDs. Indeed, a stack of quantum dots may produce a quantum object elongated and uniaxially compressed along the growth axis, which would have a light-hole like ground state and would emit preferentially in the TM mode.

Recently, the polarization properties of photoluminescence (PL) from the cleaved edge have been investigated for columnar (Kita et al. 2002, 2006; Kawaguchi et al. 2006), closelystacked (Yu et al. 1999; Anantathanasarn et al. 2006) and InGaAs-capped (Jayavel et al. 2005) self-assembled QDs. Very recently, evidence of net TM gain under optical pumping has been reported in columnar QDs (CQDs) (Kita et al. 2006), but no lasing. However, so far, no polarization studies under electrical pumping have been carried out. As edge-emitted PL may not be a reliable technique for polarization analysis due to stray light coming from the sample surface, we have measured the polarization-resolved edge-emitted electroluminescence in several QD SOA structures leading to interesting insights of QD polarization properties. The paper is organized in the following way: First in Sect. 2 the growth of QD structures and the experimental setup will be introduced. In Sects. 3 and 4 the properties of closely stackedand columnar QDs, respectively, are presented. Finally Sect. 5 compares the polarization properties of QD SOAs based on these two approaches.

\section{Experimental approach}

The active region material of our QD lasers and SOAs is based on self-assembled InAs QDs grown on GaAs substrate by molecular beam epitaxy (MBE) using either standard StranskiKrastanov (SK) growth technique or cycled submonolayer deposition. Two different growth approaches have been applied in order to realize so-called "closely-stacked-" (Yu et al. 1999) and "columnar" QDs (Kita et al. 2002; Mukai et al. 1998) which will be both introduced in the following subsections. Both approaches are based on the cycled deposition of thin InAs/GaAs layers. In the case of closely-stacked QDs the GaAs spacer layer is thick enough to produce a stack of distinct QDs - tunneling between the QDs then produces wire-like electronic states (Yu et al. 1999). In contrast, in CQDs the InAs and GaAs thickness is so small that a single Indium-rich nanostructure is formed, due to strain-driven preferential incorporation. The active region based on closely stacked or columnar QDs has been embedded between two AlGaAs cladding layers and processed into ridge waveguide laser structures of $2 \mathrm{~mm}$ length and $20-120 \mu \mathrm{m}$ width. In order to measure the polarization-resolved EL-as illustrated in Fig. 1 - the QD laser device (with as cleaved facets) has been biased with a $37 \mathrm{~Hz}$ and $50 \%$ duty cycle AC current. The edge emitted EL was then collected and collimated by a lens $\left(f_{1}=2 \mathrm{~mm}\right.$ ) and filtered by a linear polarizer (extinction ratio $>10^{5}$ in the $850-1,600 \mathrm{~nm}$ wavelength range) measuring separately TE and TM polarization by using an angle set 


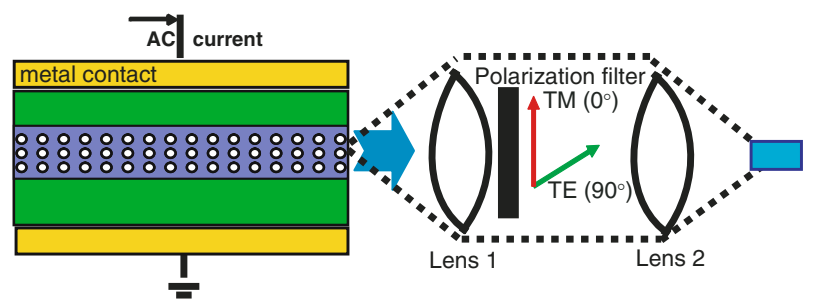

Fig. 1 Experimental setup measuring polarized and integrated electroluminescence

of $90^{\circ}$ or $0^{\circ}$, respectively. Directly after the polarizer the EL signal has been refocussed $\left(f_{2}=2 \mathrm{~mm}\right)$ on a Germanium photo detector connected to a lock-in amplifier in order to measure the integrated and polarization resolved EL. With the same experimental setup by coupling the signal after the second lens into a fiber the edge-emitted electroluminescence spectrum was measured by a optical spectrum analyzer. We note that due to the metal contacts on top of the device, luminescence coming from the sample surface is not collected, and only edge-emitted luminescence is measured.

\section{Closely-stacked quantum dots}

Several PL test samples and SOA structures were grown and processed to implement the closely-stacked QD design. The growth structure resulting in optimised PL-maximizing the ground state (GS) PL peak by varying the thickness of GaAs spacer, growth temperature and rate-is shown in Fig. 2, and consists of ten repetitions of InAs QDs with 2.4 monolayers (MLs), grown at $500^{\circ} \mathrm{C}$ and $0.085 \mathrm{ML} / \mathrm{s}$ growth rate, spaced by a GaAs layer with thickness of 4,6 or $10 \mathrm{~nm}$. After the GaAs spacer, the temperature was ramped to $580^{\circ} \mathrm{C}$ for few minutes, in order to desorb the floating In-the so-called Indium flush technique (Fafard et al. 1999). In Fig. 3 the PL spectra at room temperature of the different samples are illustrated and samples with 4-nm and 6-nm spacing reveals clearly distinguishable GS and excited state (ES) emission peaks. A strong blue shift, along with a reduction of the PL intensity, is observed for reduced spacer thickness, presumably as a consequence of the increased strain. Cross-sectional transmission electron microscopy images of the three samples were taken, and are shown in Fig. 4 for (a) 10-nm and (b) 4-nm spacing, respectively.

Fig. 2 Schematics of closely-spaced QDs

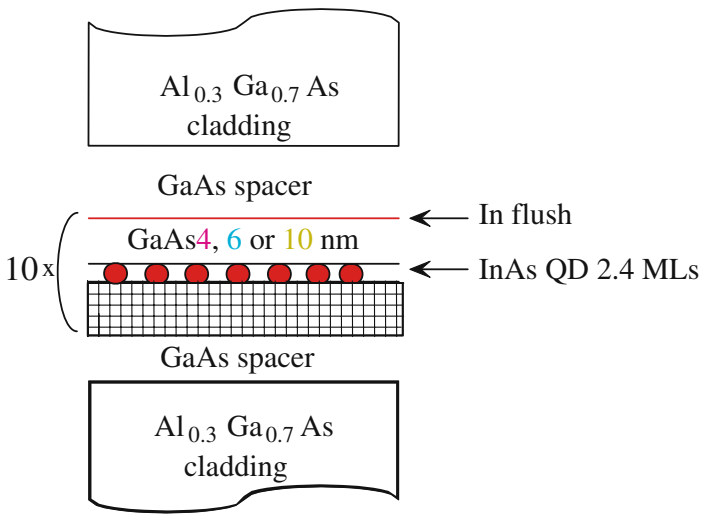




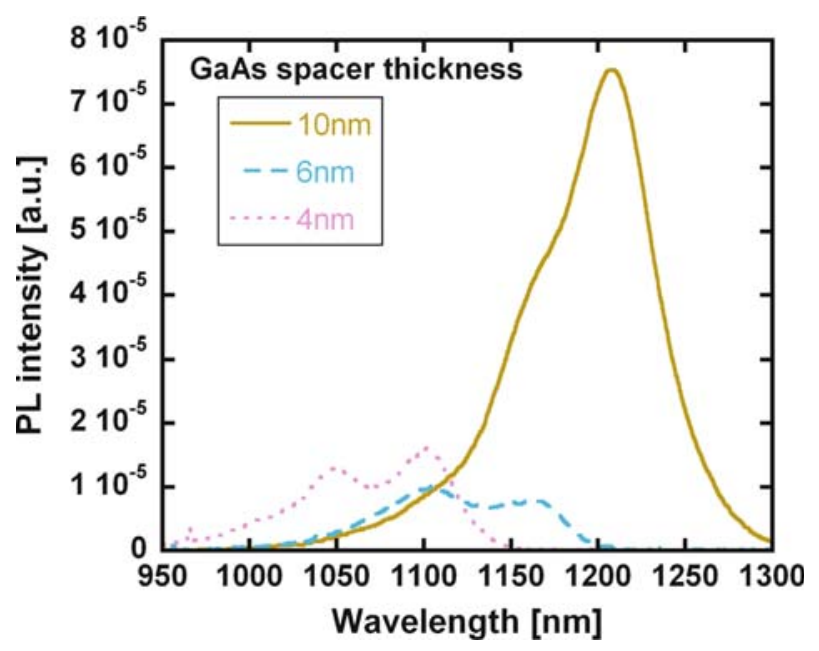

Fig. 3 PL spectra of closely-spaced QDs for different spacer thickness

Along the growth axis a good alignment of the QDs is obtained, however a relatively strong inhomogeneity of the QD dimensions is also observed. The latter is related to the growth conditions which change from layer to layer due to the varying strain state of the capping layer which may influence e.g. the critical thickness. This is expected to produce localisation of the electron wavefunction in a subset of the stack, with a negative influence on the polarisation dependence. Three SOA devices were grown and processed, each incorporating one stacked QD structure with 4,6 or $10 \mathrm{~nm}$ spacing in the active region. The edge-emitted electroluminescence (power and spectrum) from the devices was measured for the two polarisations TE and TM. In Fig. 5 the spectrum and integrated electroluminescence for each device is presented. As compared to the $10 \mathrm{~nm}$-spacing structures, the closer spacing devices present a lower efficiency and blue-shifted emission, similar to the PL spectra. However, while in the $10 \mathrm{~nm}$ spectra (Fig. 5a, top row) — as well as in standard QD multilayers with $>30 \mathrm{~nm}$ spacing —only an unstructured, broad TM emission is measured, possibly originating from deep levels in the GaAs, in 6- and 4-nm spacing samples (Fig. 5b, c, top row) clear peaks are observed for TM polarisation, as indicated by the black arrows. Furthermore, these peaks shift together with the main TE peak as the spacing is reduced, confirming that this TM emission is originated from the QD. A higher ratio of TM/TE integrated intensities is also measured for the smaller spacing samples (Fig. 5c, bottom). We thus tentatively attribute the TM peaks to emission from light-hole like excitons in the QD, which become confined as the spacing is reduced, due to the strain redistribution. Nevertheless, the energy spacing between heavy- and lighthole states is quite significant (e.g. $\approx 76 \mathrm{meV}$ for $6 \mathrm{~nm}$ spacing), which makes it difficult to populate the two states equally and obtain polarisation-independent emission. This can be traced to the spatial inhomogeneity of the QD dimension and composition observed by TEM. In summary, an increase of TM emission has been observed in closely-spaced QDs ( $4 \mathrm{~nm}$ spacing) related to a decreasing energy separation $(\approx 64 \mathrm{meV})$. However, optimisation of the growth appears challenging as the InAs thickness deposited on each layer should be separately optimised to obtain a uniform QD stack. Additionally, the PL and electroluminescence intensity measured at room temperature (RT) on these samples is rather weak, and tends to blue-shift (getting farther away from the $1,300 \mathrm{~nm}$ target) as the spacing is reduced. 

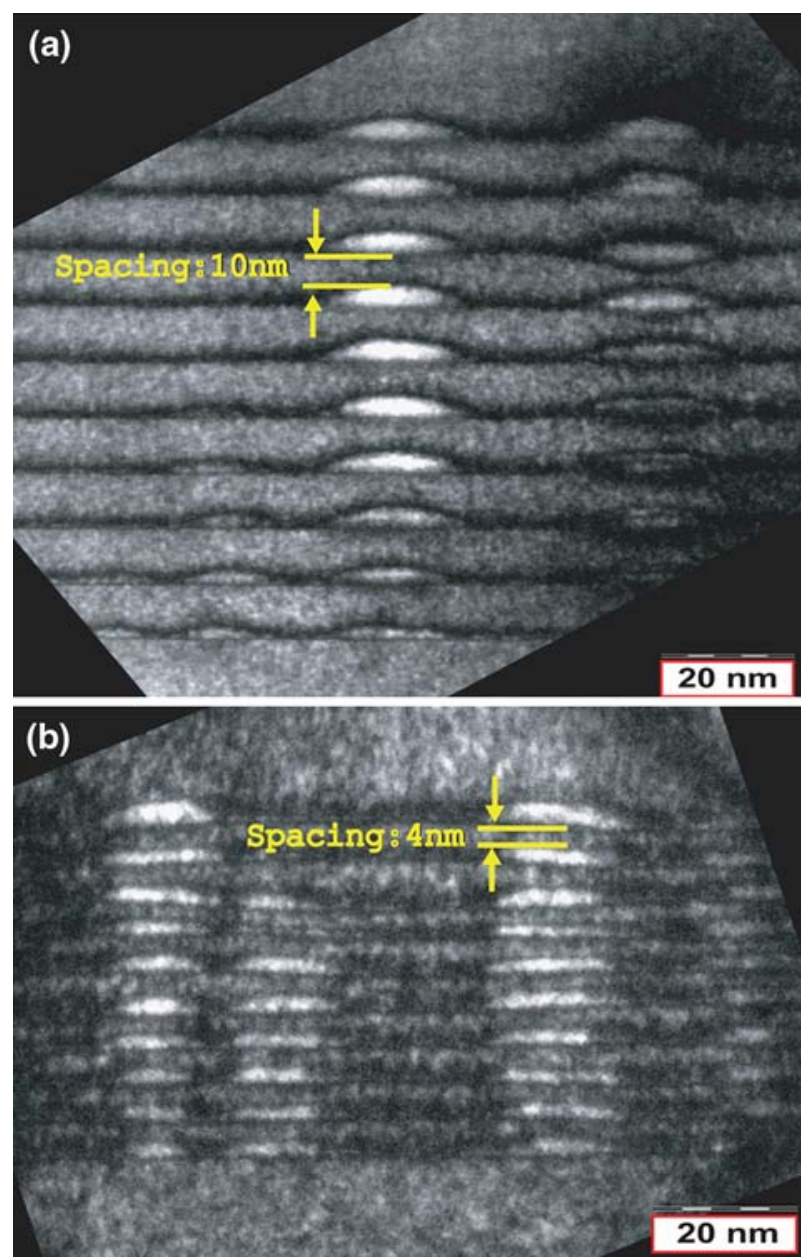

Fig. 4 TEM image of $10 \mathrm{~nm}(\mathbf{a})$ and $4 \mathrm{~nm}(\mathbf{b})$ spaced QDs

\section{Columnar quantum dots}

Columnar QDs are semiconductor nanostructures with large aspect ratio resulting from the close stacking a large number (20-30) of InAs/GaAs superlattice (SL). Due to their nearly cubic shape, they were proposed for the realisation of polarisation independence (Kita et al. 2002, 2003). As compared to the closely-stacked QDs described in the previous sections, in this case after a first layer of seed QDs, the InAs thickness deposited in each cycle is lower than the critical thickness, and the GaAs spacer is only few MLs thick so that effectively a single nanostructure is formed after the cycled InAs/GaAs deposition. As shown in Fig. 6 the growth sequence of CQDs is as follows:

$y$ MLs InAs $+N \times(3$ MLs GaAs $/ x$ MLs InAs $) /$ GaAs

A growth optimisation study was conducted, varying thickness of seed QD layer $y(1.8,1.9,2.0$ and 2.1 MLs), period of SL $N(8,10,16,24,32)$, and InAs thickness in SL $x(0.4,0.5,0.6,0.7)$. The growth conditions maximising room-temperature PL were: $y=1.8 \mathrm{MLs}, N=10$ and 

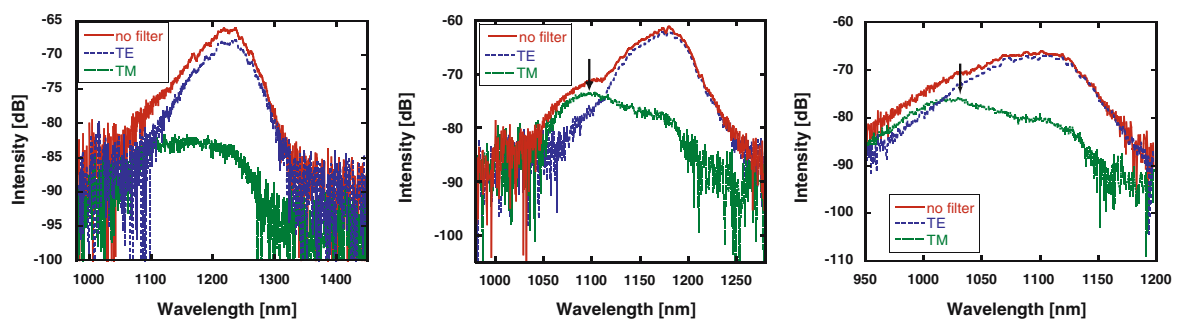

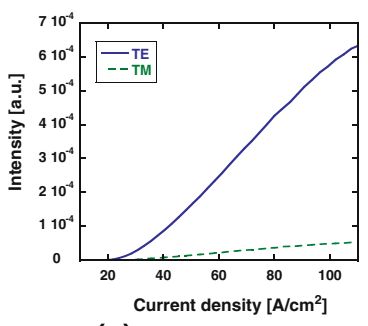

(a) $10 \mathrm{~nm}$ GaAs spacer

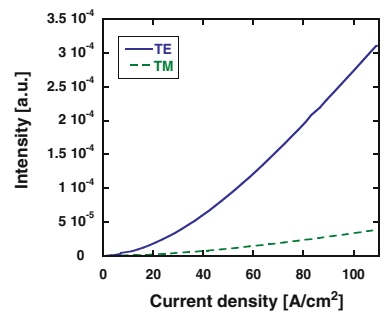

(b) $6 \mathrm{~nm}$ GaAs spacer

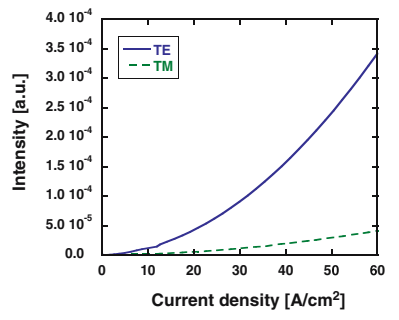

(c) $4 \mathrm{~nm}$ GaAs spacer

Fig. 5 Spectrum (top row) and corresponding integrated electroluminescence (bottom row) of closely stacked QDs with 10-, 6- and 4 nm GaAs spacing

Fig. 6 Schematics of columnar QD growth

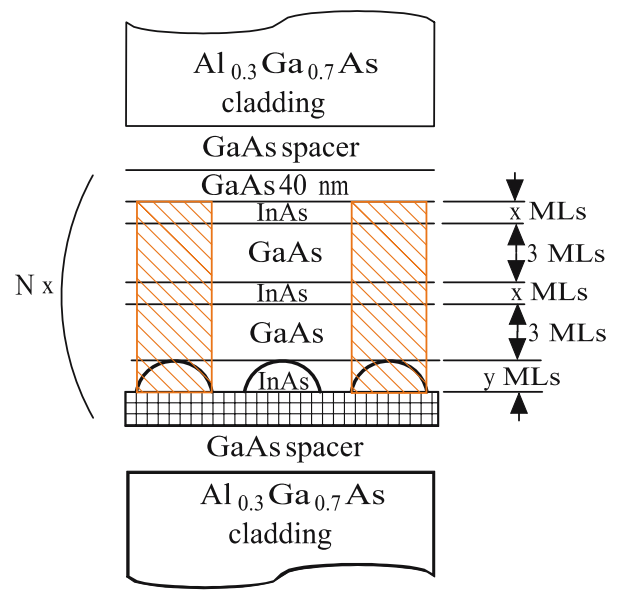

$x=0.7 \mathrm{MLs}$. The growth temperature was $515^{\circ} \mathrm{C}$, and the growth rate $0.1 \mathrm{ML} / \mathrm{s}$. The formation of QDs was confirmed by transmission electron microscopy images (Fig. 7), showing Indium-rich islands, much taller than usual Stranski-Krastanov QDs and with nearly cubic shape, as expected-embedded in a QW structure (dark region). The Indium composition profile (Fig. 8) along the growth axis (red arrow in Fig. 7) of a columnar QD (solid line) and compared to the adjoining QW structure (dashed line)-indicated an Indium content varying from $25 \%$ (center) to $35 \%$ (top and bottom), which may induce a localisation of the wavefunction in the Indium-rich regions. It should be noted that these QDs represent a very peculiar type of nanostructure as compared to standard SK QDs. They may have interesting properties, besides polarisation dependence, for laser applications. The PL spectra (Fig. 9) of a columnar QD having $y=1.8 \mathrm{MLs}, N=10$ and $x=0.7$ for $17 \mathrm{~mW}$ (dashed line) and $50 \mathrm{~mW}$ (solid) pump power shows several peaks in the 1,000-1,100 nm wavelength 


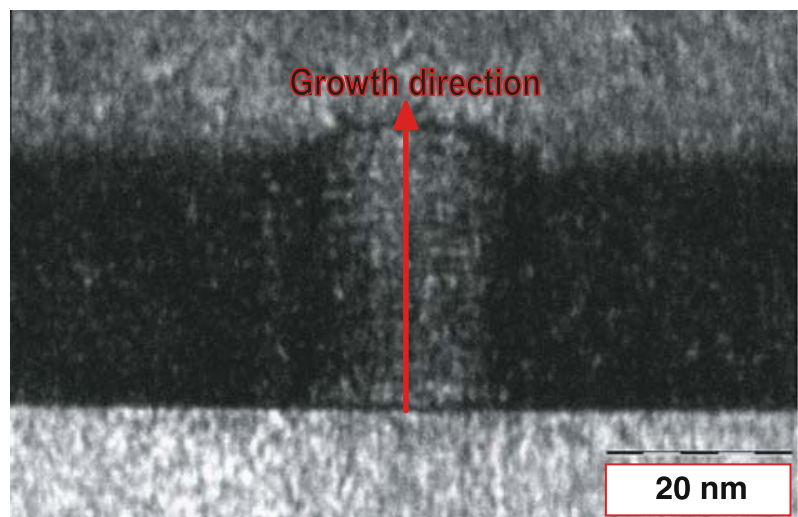

Fig. 7 TEM image of columnar QDs

Fig. 8 Indium profile of CQDs compared to adjoining QW structure along growth axis

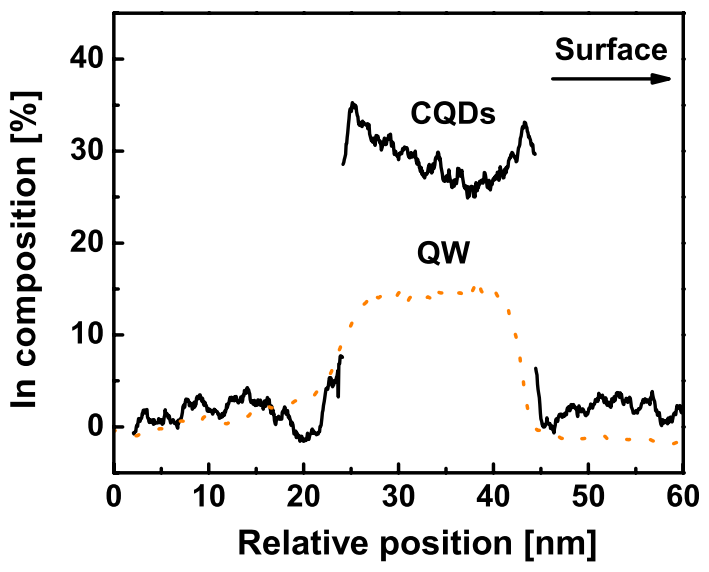

region, which can be attributed to several states in the QDs. The state-filling behavior observed for different pump powers along with previous detailed characterization and modeling (Motyka et al. 2007) allows us to evidence emissions from the 2D QW, excited- (ES) and ground-state (GS). Based on this active material, SOA structures were grown and processed, incorporating five layers of columnar QDs obtained by depositing 1.8 MLs InAs seed layer and then 10 or 18 cycles of $3 \mathrm{ML} \mathrm{GaAs} / 0.62 \mathrm{ML}$ InAs. An increased TM/TE ratio was observed - as compared to conventional 1,300 nm-emission InAs QDs (obtained by depositing a single layer of InAs and capping with an InGaAs layer) (Fig. 10a) - as shown in the polarisation-resolved TE and TM emission spectra and integrated intensities for 10- and 18-cycles (Fig. 10b, c), respectively. In the case of 18-cycles QDs, the TM emission peak is only $25 \mathrm{~nm}$ blue-shifted as compared to TE, and the integrated intensity is only a factor of two lower-a large improvement over standard QDs. We note that InGaAs-capped single-layer QDs in Fig. 10a—differently from the uncapped QDs in Fig. 5a—exhibit a TM emission peak at the GS wavelength of $1,270 \mathrm{~nm}$. We attribute this to a larger light-hole component of the GS wavefunction due to the modified QD shape. Nevertheless, the integrated TM emission remains very low in single-layer QDs. 
Fig. 9 PL spectra of CQDs at room temperature for two different pump powers
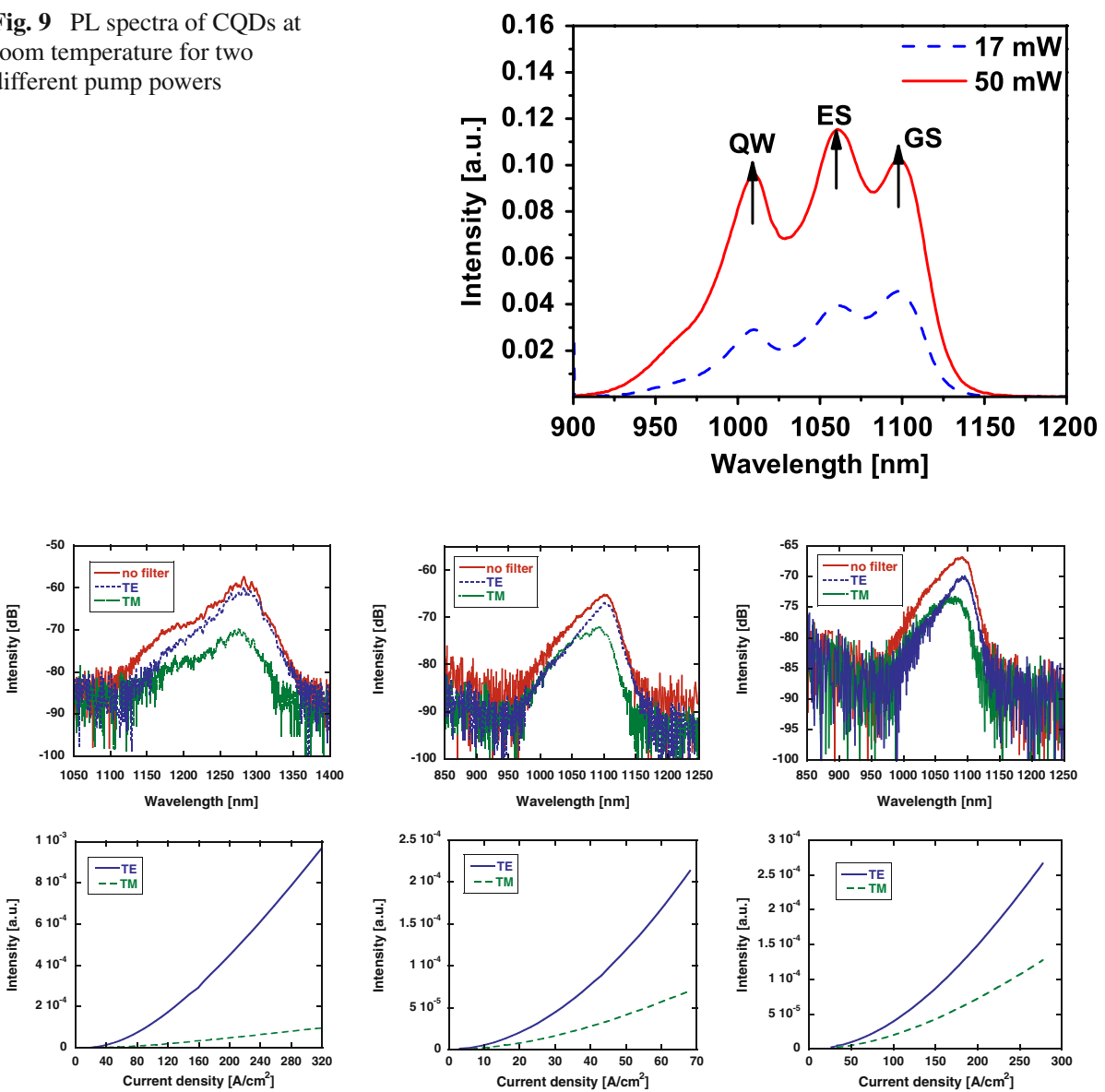

(a) Standard QDs

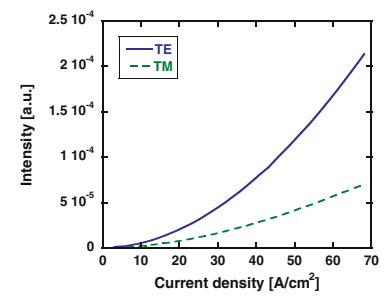

(b) Columnar QDs (10 cycles)

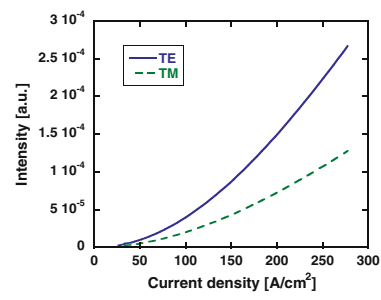

(C) Columnar QDs (18 cycles)

Fig. 10 Polarisation-resolved (TE/TM) integrated intensities (top row) and emission spectra (bottom row) of standard QDs, columnar QD with 10- and 18 cycles

\section{Comparison of the polarization properties}

In order to facilitate a quantitative comparison between the different approaches, Fig. 11 reports the ratio of TM and TE integrated electroluminescence intensities from SOAs incorporating columnar (10- and 18 cycles), closely-stacked (4-, 6- and $10 \mathrm{~nm} \mathrm{GaAs} \mathrm{spacer),}$ and standard $40 \mathrm{~nm}$-spaced QDs. While closely-stacked QDs represent a small improvement over standard (uncoupled) QDs—due to its not uniform QD stacking—a clear improvement is observed in columnar QDs with a large number of deposition cycles. The comparison of the polarisation-resolved (TE/TM) emission spectra (Fig. 5 and Fig. 10) in terms of the smallest energy spacing in closely-stacked QDs with $4 \mathrm{~nm} \mathrm{GaAs}$ spacer ( $\approx 64 \mathrm{meV})$ and 18 cycles columnar QDs $(\approx 21 \mathrm{meV})$, respectively, confirms also the potential of CQDs towards a polarization-independent active material for SOAs.

On the other side, the residual polarization-dependence in columnar QDs can be likely linked to a inhomogeneous indium distribution in the QD - as shown before in Fig. 8 - and 
Fig. 11 TM/TE ratio of integrated intensities for SOAs with different active regions: columnar (10- and 18 cycles), closely stacked (4-, 6- and $10 \mathrm{~nm}$ GaAs spacer), and standard 40nm-spaced QDs

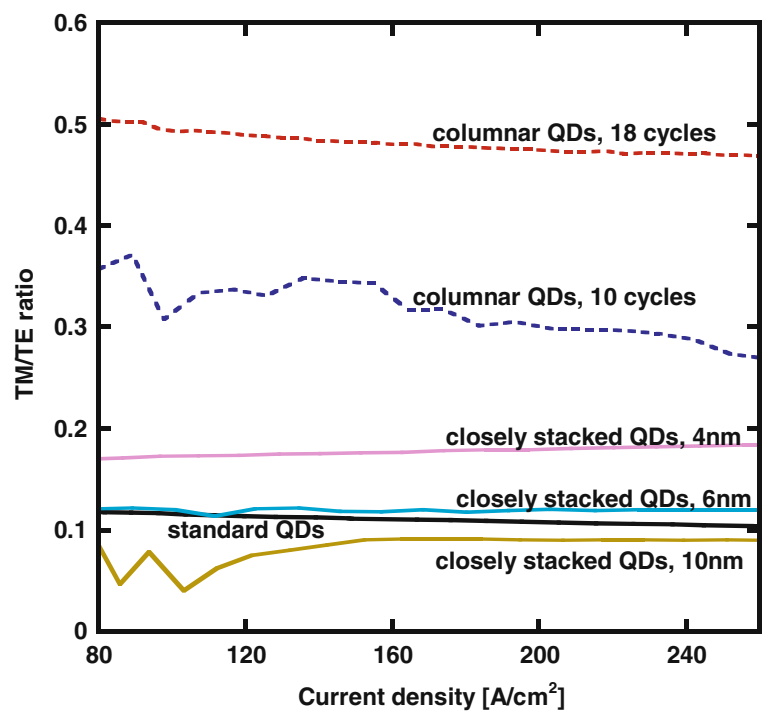

to the residual compressive strain which could be responsible that heavy-and light hole-like states are still energetically separated.

\section{Conclusions}

In summary, we have applied QD shape engineering in order to control the optical polarization anisotropy of QDs. We have fabricated and tested several SOA devices based on closelystacked QDs and CQDs as presented in previous sections. In closely-stacked QDs we observed a good alignment of the QDs along the growth axis, but also a relatively strong inhomogeneity of the QD dimensions related to varying capping layer strain state during the growth. As a consequence we are expecting that localization of the electron wavefunction in QD stack's subset will be produced, with a negative influence on the polarization dependence. Analyzing the polarization-resovled edge-emitted EL of closely-stacked QD SOA devices we observe a small increase of TM emission in closely-spaced QDs combined with a decreasing energy separation. In addition, we observed very weak PL and electroluminescence intensities which tends to blue-shift as the spacing is reduced and getting farther away from the $1,300 \mathrm{~nm}$ target.

On the other side columnar QDs present a nearly cubic shape much different from ordinary Stranski-Krastanov QDs. The Indium content profile might induce localization of the wavefunctions and will be crucial for controlling the polarization's anisotropy. From polarized edge-emitted EL — measuring both integrated intensity and spectra—we observed a very good improvement of TM/TE ratio combined with small spectral energy spacing compared to standard and closely-stacked QDs. Although there is still a residual polarization dependence in our columnar QDs which is related to the non-uniform Indium profile, and to anisotropic strain distribution, we expect a promising potential for such kind of QD structures in future SOA devices.

Acknowledgements We acknowledge useful discussions with G. Sek, J. Andrzejewski and J. Misiewicz (Wroclaw University of Technology, Poland). This work has been supported by EU-FP6 project ZODIAC (contract number FP6/017140), the OFES-COST program, and the Swiss National Science Foundation. 


\section{References}

Akiyama, T. et al.: An ultrawide-band semiconductor optical amplifier having an extremely high penalty-free output power of $23 \mathrm{dBm}$ achieved with quantum dots. IEEE Photon. Tech. Lett. 17, 1614-1616 (2005)

Anantathanasarn, S. et al.: Stacking and polarization control of wavelength-tunable (1.55 $\mu \mathrm{m}$ region) InAs/InGaAsP/InP (100) quantum dots. Appl. Phys. Lett. 88, 063105 (2006)

Berg, T.W. et al.: Quantum dot amplifiers with high output power and low noise. Appl. Phys. Lett. 82, 30833085 (2003)

Fafard, S. et al.: Lasing in quantum-dot ensembles with sharp adjustable electronic shells. Appl. Phys. Lett. 75, 986-988 (1999)

Jayavel, P. et al.: Optical polarization properties of InAs/GaAs quantum dot semiconductor optical amplifier. Jpn. J. Appl. Phys. 44, 2528-2530 (2005)

Kawaguchi, K. et al.: Controlling polarization of $1.55 \mu \mathrm{m}$ columnar InAs quantum dots with highly tensilestrained InGaAsP barriers on InP(001). Jpn. J. Appl. Phys. 45, L1244-L1246 (2006)

Kita, T. et al.: Polarization-independent photoluminescence from columnar InAs/GaAs self-assembled quantum dots. Jpn. J. Appl. Phys. 41, L1143-L1145 (2002)

Kita, T. et al.: Polarization controlled edge emission from columnar InAs/GaAs self-assembled quantum dots. Phys. Stat. Sol. (c) 0, 1137-1140 (2003)

Kita, T. et al:: Artificial control of optical gain polarization by stacking quantum dot layers. App. Phys. Lett. 88, 211106 (2006)

Motyka, M. et al.: Optical and electronic properties of GaAs-based structures with columnar quantum dots. Appl. Phys. Lett. 90, 181933 (2007)

Mukai, K. et al.: Lasing with low threshold current and high output power from columnar-shaped InAs/GaAs quantum dots. Electron. Lett. 34, 1588-1589 (1998)

Sugawara, M. et al.: Quantum-dot semiconductor optical amplifiers for high bit-rate signal processing over 40 Gbit/s. Jpn. J. Appl. Phys. 40, L488-L491 (2001)

Yu, P. et al.: Optical anisotropy in vertically coupled quantum dots. Phys. Rev. B 60, 16680-16685 (1999) 